\title{
Antenna Gain and Scattering Measurement Using Reflective Three-Antenna Method
}

\author{
Hsin-Chia Lu and Tah-Hsiung Chu* \\ Department of Electrical Engineering, \\ National Taiwan University, Taipei, Taiwan, R.O.C. \\ E-mail:leonardo@ew.ee.ntu.edu.tw, thc@ew.ee.ntu.edu.tw
}

\section{INTRODUCTION}

Three-antenna method [1] is a technique to measure the antenna gain without using a reference antenna. Since it operates in a transmission-type arrangement, a long return cable is required. Several methods were then developed to eliminate the return cable by using the radar cross section (RCS) measurement method. The advantages of this method are described in [2]-[6]. In [7], a measurement method based on RCS measurement concept and antenna scattering matrix is developed. It can not only measure the antenna gain, but also derive the structural scattering characteristics and antenna input impedance from the measurement. However, it requires a complicated polarimetric calibration procedure for the measurement system

In this paper, a novel method is proposed to combine the concepts of three-antenna method and RCS measurement method to measure the antenna gain and its structural scattering characteristics without involving the reference antenna or polarimetric calibration. In this method, the measurement arrangement including the transmitting and receiving antennas is considered as a two-port network with reference planes at the input port for each antenna as shown in Fig.1. By connecting three different known terminators at the receiving antenna port and measure the reflection coefficients at the transmitting antenna port, the scattering parameters of this two-port network can be derived. They are shown to be related to the product of two antennas' gain. Therefore one can follow the three-antenna method to find the gain of each antenna. In addition, the structural scattering characteristics of each antenna can be solved.

\section{FORMULATION}

As shown in Fig.1, antenna $i$ and antenna $j$ and the free space between them can be considered as a two-port network with its reference planes at the terminating ports $i$ and $j$. The two-port scattering matrix $\left[S^{T}\right]$ of this two-port network is then related to the scattering matrices $\left[S^{i}\right]$ and $\left[S^{j}\right]$ of each antenna. In the following section, the formulation to derive the two-port scattering matrix $\left[S^{T}\right]$ using the reflection meas urement is given first. The relation between the measured two-port scattering parameters $\left[S^{T}\right]$ and two antenna scattering matrices is given in Sec. II.B. The concept of 
three-antenna method is then adopted to measure the antenna gain and its structural scattering from the antenna scattering matrix representation.

A. Two-port scattering parameter measurement using reflection measurement As a terminator with reflection coefficient of $\Gamma_{a}$ is connected at the port 2 of a reciprocal two-port network, $S_{11}^{(a)}$ of this terminated one-port network is related to $S_{i j}$ of the original two-port network is

$$
S_{11}^{(a)}=S_{11}+\frac{S_{12}^{2} \Gamma_{a}}{1-S_{22} \Gamma_{a}} .
$$

By connecting three known different terminators $\Gamma_{a}, \Gamma_{b}$ and $\Gamma_{c}$ at port 2, (1) becomes

$$
\begin{aligned}
& S_{11}^{(a)}=S_{11}+\frac{S_{12}^{2} \Gamma_{a}}{1-S_{22} \Gamma_{a}}, \\
& S_{11}^{(b)}=S_{11}+\frac{S_{12}^{2} \Gamma_{b}}{1-S_{22} \Gamma_{b}}, \\
& S_{11}^{(c)}=S_{11}+\frac{S_{12}^{2} \Gamma_{c}}{1-S_{22} \Gamma_{c}} .
\end{aligned}
$$

By equating $S_{12}^{2}$ at the right hand side in (2) to (4), one can obtain two linear equations of $S_{11}$ and $S_{22}$ as

$$
\begin{aligned}
& \left(\frac{1}{\Gamma_{a}}-\frac{1}{\Gamma_{b}}\right) S_{11}+\left(S_{11}^{(a)}-S_{11}^{(b)}\right) s_{22}=\frac{s_{11}^{(a)}}{\Gamma_{a}}-\frac{S_{11}^{(b)}}{\Gamma_{b}}, \\
& \left(\frac{1}{\Gamma_{a}}-\frac{1}{\Gamma_{c}}\right) S_{11}+\left(S_{11}^{(a)}-S_{11}^{(c)}\right) S_{22}=\frac{S_{11}^{(a)}}{\Gamma_{a}}-\frac{S_{11}^{(c)}}{\Gamma_{c}} .
\end{aligned}
$$

From (5) and (6), $S_{11}$ and $S_{22}$ can be solved. In addition, $S_{12}^{2}$ can be calculated by substituting the resulted $S_{11}$ and $S_{22}$ into (2) to (4). Note that the sign of $S_{12}$ is ambiguous.

\section{B. Reflective three-antenna method}

In the following derivation, each antenna is represented as a two-port network, denoted by its terminating port and radiation port as shown in Fig.1. This two-port scattering matrix describes the antenna input impedance, structural scattering and transmitting and receiving characteristics as illustrated in [7]. Taking antenna $i$ in Fig. 1 for example, it is given as

$$
\left[\begin{array}{l}
b_{1}^{i} \\
b_{2}^{i}
\end{array}\right]=\left[\begin{array}{ll}
s_{11}^{i} & s_{12}^{i} \\
s_{21}^{i} & s_{22}^{i}
\end{array}\right]\left[\begin{array}{l}
a_{1}^{i} \\
a_{2}^{i}
\end{array}\right]
$$


$S_{i i}$ is its input impedance, $S_{12}^{i}=S_{21}^{i}$ accounts for the antenna transmitting or receiving characteristics, and $S_{22}^{i}$ describes the antenna structural scattering characteristics. Based on the formulation given in Sec. II.A, the scattering matrix $\left[S^{T}\right]$ can be calculated from three reflection measurements by terminating port $j$ with three different ter minators. [ $S^{T}$ ] is a cascade of three scattering matrices to account for the transmitting antenna $i$, free space propagation and the receiving antenna $j$, and it is given as

$$
\begin{gathered}
S_{11}^{T}=S_{11}^{i}+\frac{\left(S_{12}^{i}\right)^{2} S_{22}^{j} T^{2}}{1-S_{22}^{i} S_{22}^{j} T^{2}}, \\
S_{22}^{T}=S_{11}^{j}+\frac{\left(S_{12}^{j}\right)^{2} S_{22}^{i} T^{2}}{1-S_{22}^{i} S_{22}^{j} T^{2}}, \\
S_{12}^{T}=\frac{S_{12}^{i} S_{12}^{j} T}{1-S_{22}^{i} S_{22}^{j} T^{2}},
\end{gathered}
$$

where $T$ is the range term

As the input impedance of each antennas is measured in advance, one can calculate $\Delta S_{11}$ and $\Delta S_{22}$ as $\Delta S_{11}=S_{11}^{T}-S_{11}^{i}$ and $\Delta S_{22}=S_{22}^{T}-S_{11}^{j}$. The product of transmitting characteristics and structural scattering of $i$ and $j$ antennas are then given as

$$
\begin{gathered}
S_{22}^{i} S_{22}^{j}=\frac{\Delta S_{11} \Delta S_{22}}{\left(S_{12}^{T}\right)^{2} T^{2}}, \\
S_{12}^{i} S_{12}^{j}=S_{12}^{T} \frac{\left(1-S_{22}^{i} S_{22}^{j} T^{2}\right)}{T} .
\end{gathered}
$$

Note that although $S_{12}^{T}$ has a sign ambiguity problem given in Sec. II.A, the calculation of (11) is well determined.

By using the concept of three-antenna method, one can solve the transmitting characteristics of each antenna from the measured response of three pairs of three different antennas. As $S_{12}^{i}$ is solved, the maximum available antenna gain $G_{i}^{m}$ and the transducer antenna gain $G_{i}^{l}$ of the antenna $i$ can be calculated as

$$
G_{i}^{m}=\frac{\sqrt{4 \pi}}{\lambda} \frac{\left|S_{12}^{i}\right|^{2}}{1-\left|S_{11}^{i}\right|^{2}} \text { and } G_{i}^{i}=\frac{\sqrt{4 \pi}}{\lambda}\left|S_{12}^{i}\right|^{2} \text {. }
$$

Since only the absolute value of $s_{12}^{i}$ is involved in the calculation of antenna gain, its sign ambiguity is not important. The structural scattering characteristics of antenna $i$ is then given as

$$
S_{22}^{i}=\Delta S_{22} \frac{1-S_{22}^{i} S_{22}^{j} T^{2}}{\left(S_{12}^{j}\right)^{2} T^{2}} .
$$

Note there is no sign ambiguity problem in (14) 


\section{CONCLUSION}

In this paper, we proposed a novel method to measure the antenna gain and structural scattering characteristics using a reflective three-antenna method. This method can eliminate the needs of a return cable, reference antenna or the polarimetric calibration procedure.

\section{REFERENCE}

[1] J. S. Hollis, T. J. Lyon and L. Clayton, Microwave Antenna Measurements, Ch.8, Scientific-Atlanta Inc. 1970.

[2] D. D. King, "Measurement and interpretaion of antenna scattering," Proc. IRE., vol. 37, pp. $770-777$, July 1949.

3] R. J. Garbacz, "Determination of antenna parameters by scattering cross section measurements," Proc. Inst. Elect. Eng., vol. 111, no. 10, pp. 1679-1686, Oct. 1964.

[4] J. Appel-Hansen, "Accurate determination of gain and radiation patterns by radar crosssection measurements," IEEE Trans. Antennas Propagat., vol. AP-27, pp. 640-646, Sept. 1979.

[5] J. J. H. Wang, C. W. Choi and R. L. Moore, "Precession experimental characterization of the scattering and radiation properties of antennas," IEEE Trans. Antennos Propagat., vol. AP-30, pp. 108-112, Jan. 1982.

[6] K. M. Lambert, R. C. Rudduck and T. H. Lee, "A new method for obtaining antenna gain from backscatter measurements," IEEE Trans. Antennas Propagar., vol. AP-38, pp.896902 , June 1990 .

[7] W. Wiesbeck and E. Heidrich, "Wide-band multiport antenna characteriztion by polarimetric RCS measurement," IEEE Trans. Antennas Propagat., vol. AP-46, pp. 341-350, March 1998.
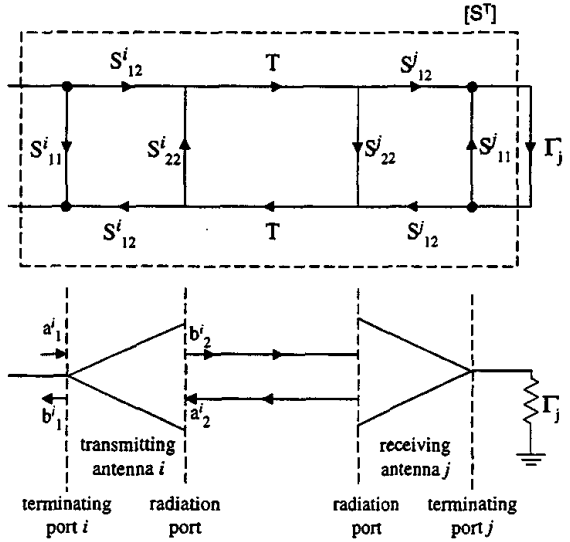

Fig. 1 Scattering parameter representaion for the reflective three-antenna method. 